The adenocarcinoma detection rate was $2.8 \%$ (6) and all of them had had either repeat scope at the 3 and 12 months intervals or had surgery. $12(5.6 \%)$ had tubular/tubulovillous adenoma with high grade dysplasia. $8(66 \%)$ of these patients had repeat scope at 3 months, whilst 1 (8\%) underwent surgery and $3(25 \%)$ made an informed choice to not have further colonoscopic examination.

\begin{tabular}{|c|c|c|c|c|c|c|}
\hline Complications & Caecum & $\begin{array}{l}\text { Ascending } \\
\text { colon }\end{array}$ & $\begin{array}{l}\text { Transverse } \\
\text { colon }\end{array}$ & $\begin{array}{l}\text { Descending } \\
\text { colon }\end{array}$ & $\begin{array}{l}\text { Sigmoid } \\
\text { colon }\end{array}$ & Rectum \\
\hline $\begin{array}{l}\text { Immediate } \\
\text { bleeding }\end{array}$ & 3 & 0 & 2 & 0 & 2 & 4 \\
\hline $\begin{array}{l}\text { Delayed } \\
\text { bleeding }\end{array}$ & 0 & 0 & 0 & 0 & 0 & 0 \\
\hline Perforation & 0 & 1 & 0 & 0 & 0 & 0 \\
\hline $\begin{array}{l}\text { Recurrence at } \\
3 / 12\end{array}$ & 0 & 0 & 0 & 0 & 0 & 3 \\
\hline
\end{tabular}

Our rescope rate for the polyp size $\geq 20 \mathrm{~mm}$ at 3 months and 12 months was $69 \%$ and $78 \%$ respectively. The main reasons for not having the scope were either patient choice or having surgery/other forms of cancer treatment.

Conclusions EMR is an effective and safe approach in the expert hands for the management of colonic polyps. The role of prophylactic endoclips is still unclear in reducing the risk of post-procedural bleeding and it is highly dependent on the operator's preference and experience.

Total character count 2817.

\section{PTH-021 CANCER AND ADENOMA DETECTION RATE IN 2-WEEK WAIT COLONOSCOPY AND CT COLONOGRAPHY- TERTIARY CENTRE EXPERIENCE}

\footnotetext{
${ }^{1}$ Sardar Chaudhary*, ${ }^{2}$ Jay Pancholi, ${ }^{2}$ Catalin Ivan, ${ }^{2}$ Ratan Verma, ${ }^{2}$ Mosheir Elabassy, ${ }^{1}$ Richard J Robinson, ${ }^{2} J a m e s$ A Stephenson. 'Digestive Diseases Centre, UHL, Leicester, UK; ${ }^{2}$ Gastrointestinal Imaging Group, UHL, Leicester, UK
}

\subsection{6/gutjnl-2018-BSGAbstracts.43}

Introduction Computed Tomography Colonoscopy (CTC) is increasingly being used as an alternative to optical colonoscopy (OC). The SIGGAR trial and COCOS study have shown CTC to be an accurate and safe alternative to OC for diagnosis of colorectal cancer (CRC) and large polyps. Our aim was to compare various performance indicators between CTC and OC in the lower GI straight to test (STT) 2 week wait (2 WW) pathway.

Methods The investigation of choice for the lower GI 2 WW STT cohort between Nov 2014 and Oct 2015 was OC with CTC being the first-line test between Jan 2016 and Dec 2016. We retrospectively analysed 12 months' data for both cohorts. Outcomes included completion rate, polyp detection rate (PDR), adenoma detection rate (ADR) and CRC detection rates.

Results 1135 patients attended for OC versus 1829 for CTC. Significantly more OC were cancelled on the day compared to CTC $(6.5 \% \mathrm{v} 2 \% \mathrm{p}=0.0001)$. OC had a completion rate measured by caecal intubation of $86 \%$ versus $100 \%$ of CTC $(\mathrm{p}=0.0001)$. The diagnostic study rate measured by adequate bowel preparation/faecal tagging and distension was $89.3 \%$ at OC (adequate preparation) versus $98.4 \%$ for CTC $(\mathrm{p}=0.0001)$.

CRC detection rate was $4.5 \%$ (95\% CI $3.43 \%$ to $5.95 \%$ ) in the OC group versus $4.9 \%$ (95\% CI $3.97 \%$ to $5.95 \%)$ in the CTC group $(p=>0.005)$.

The PDR was significantly higher in the OC group compared to the CTC group $(25.1 \% \quad(95 \%$ CI $22.95 \%$ to $27.32 \%)$ v $13.5 \%$ (95\% CI $11.95 \%$ to $15.11 \%) \mathrm{p}=0.0001)$. However, $61.7 \%$ of the polyps detected at OC were $\leq 5 \mathrm{~mm}$ which are not routinely reported on CTC. PDR for polyps $>5$ $\mathrm{mm}$ at OC was $9.6 \%$ (95\% CI $7.98 \%$ to $11.54 \%)$ versus $13.5 \%$ at CTC $(p=0.0024)$.

The ADR at OC was significantly higher than in the CTC (16.5\% (95\% CI $14.38 \%$ to $18.85 \%$ ) versus $9.8 \%$ (95\% CI $8.53 \%$ to $11.29 \%) \mathrm{p}=0.0001)$.

The non-CRC detection rate in the CTC group was $4.3 \%$. Conclusions CTC and OC have comparable CRC detection rates for patients referred via the 2 WW STT lower GI pathway. However, the PDR and ADR are higher with OC, this data is skewed by the number of diminutive polyps identified at OC that are not routinely reported at CTC. Also, a number of cases with $5-10 \mathrm{~mm}$ polyps at CTC did not progress to OC. Long term follow up data is required to compare interval cancer detection rates in these cohorts to assess the impact of not reporting/identifying sub $5 \mathrm{~mm}$ polyps.

From a service delivery perspective, the on the day cancellation rate, completion rate and diagnostic study rate for CTC were significantly better than OC in the STT patient.

An additional benefit of CTC is the diagnosis of noncolonic cancers, the rate of which was comparable to the CRC rate.

\section{PTH-022 HYPERAMYLASAEMIA POST ANTEGRADE DOUBLE BALLOON ENTEROSCOPY - DOES INDOMETHACIN MAKE A DIFFERENCE?}

Stefania Chetcuti Zammit*, Suneil A Raju, Hey-Long Ching, David S Sanders, Reena Sidhu. Sheffield Teaching Hospitals, Sheffield, UK

\subsection{6/gutjnl-2018-BSGAbstracts.44}

Introduction High amylase does not always signify acute pancreatitis and it can occur due to focal areas of ischaemia in the pancreas due to mechanical stress during double balloon enteroscopy (DBE). The use of rectal NSAIDs to prevent post DBE pancreatitis has never been explored unlike in ERCP where patients receiving rectal NSAIDs have a lower incidence of pancreatitis.

Methods Patients who received rectal indomethacin (100 mg) $30 \mathrm{~min}$ prior to antegrade DBE were compared to a control group who did not receive indomethacin before the above protocol was implemented. Serum amylase and CRP 3 hours before and after DBE were compared.

Results 240 patients (56 indomethacin, 184 controls; 50\% males; mean age 58.5 \pm SD14.0) were included.

Indications included IDA (37.5\%), obscure overt gastrointestinal bleeding (17.1\%), suspected crohn's disease and strictures (17.9\%), complication of coeliac disease (1.3\%), small bowel (SB) tumours/polyps (17.9\%), others $(8.3 \%)$.

Patients had a median of $13.0 \pm \mathrm{SD} 13.0$ passes, 65.0 $\pm \mathrm{SD} 25.0 \mathrm{~min}, \quad 170 \pm \mathrm{SD} 52.0 \mathrm{~cm}$ of $\mathrm{SB}$ examined. $36.3 \%$ underwent a therapeutic procedure during DBE: APC/ 